\title{
Ion exchange accompanied by neutralization in an anion bed
}

\author{
Henry K.S. Tan \\ Department of Applied Chemistry, National Chiao Tung University, Hsinchu, Taiwan, ROC
}

Received in revised form 28 May 2002; accepted 14 June 2002

\begin{abstract}
The performance of the anion bed in a two-bed deionization process was investigated. Experiments and simulation were carried out with a feed solution concentration of about $0.005 \mathrm{~N}$. The prepared solutions were dilute acid of $\mathrm{HCl}_{2} \mathrm{H}_{2} \mathrm{SO}_{4}$ and a mixture of the two. The bed height was about $40 \mathrm{~cm}$ and the flow rate in the range of $75-150 \mathrm{ml} / \mathrm{min}$. Both the binary and ternary models were used to predict the column performance. In both models, it was found that the equilibrium parameters do not affect significantly the breakthrough behavior. This is due to the accompanied neutralization reaction which results in the irreversible form of the rate equation. The experimental and theoretical breakthrough curves show very good agreement especially for the early part of the data. Results of this study suggest that a pseudo-binary or pseudo-ternary model can be used to model the fixed bed deionization process by ion exchange.
\end{abstract}

(C) 2002 Elsevier Science B.V. All rights reserved.

Keywords: Anion exchange; Resin bed; Neutralization

\section{Introduction}

The demand for deionized water is increasing due to the rapid expansion of the electronic and microelectronic industries. Recent studies have been focused on the production of ultrapure water by ion exchange process [1,2]. Deionized water is produced using either a mixed-bed or a two-bed ion exchange system [3-10]. In the later configuration, the effluent from the cation bed is essentially a mixture of dilute acids. This effluent, when passed through the anion bed of $\mathrm{OH}^{-}$form, yields water with very low ionic content. The reaction that takes place in the anion bed not only deals with ordinary ion exchange, but also involves neutralization. Although a mixed bed has much higher treatment efficiency than a two-bed system, there are instances when a two-bed unit can be used in series with a mixed bed. When the feed water ionic concentration is high, the two-bed system can be used as a pre-treatment unit. In this case, the mixed bed unit that treats effluent from the two-bed system can produce water of utmost purity. The performance of the cation bed in the two-bed system is dictated by the same basic principles for any ion exchange column operation. However, for the anion bed, the assumption of a constant ionic concentration in the solution phase is no longer valid. This is because of the neutralization that takes place as the anion resin bed treats the effluent discharged from the cation bed. The total

E-mail address: hentan@cc.nctu.edu.tw (H.K.S. Tan) ionic concentration of the solution from an anion bed will be reduced as exchange proceeds. In this work, we present both experimental data and a simple and practical quantitative model for investigating column dynamics of anion exchange accompanied by neutralization. Simulation results were used to compare with the experimental data obtained in this study.

\section{Theoretical}

It is assumed that the feed to an anion bed is a mixture of dilute strong acids consisting of $\mathrm{HCl}$ and $\mathrm{H}_{2} \mathrm{SO}_{4}$. The continuity equations for anionic species $\mathrm{Cl}^{-}$and $\mathrm{SO}_{4}{ }^{2-}$, after neglecting axial dispersion terms, are

$u \frac{\partial C_{i}}{\partial z}+\beta \frac{\partial C_{i}}{\partial t}+\frac{\partial q_{i}}{\partial t}=0, \quad i=1,2$

where $C$ and $q$ denote the concentration of chloride or sulfate ion in the solution and resin, respectively, $z$ the axial bed distance from solution inlet, $u$ the superficial linear flow rate, $\beta$ the void fraction of the bed and $t$ the absolute time.

Since the feed is dilute, a rough estimate using the Helfferich number criterion [11] indicates that film diffusion is likely the rate-controlling step. The linear driving force rate equations based on film diffusion rate control are

$\frac{\partial q_{i}}{\partial t}=K_{\mathrm{f}} a_{\mathrm{p}}\left(C_{i}-C_{i}^{*}\right), \quad i=1,2$ 


\begin{tabular}{|c|c|}
\hline \multicolumn{2}{|c|}{ Nomenclature } \\
\hline$a_{\mathrm{p}}$ & $\begin{array}{l}\text { mass transfer surface area per unit volume of } \\
\text { contacting volume }\left(\mathrm{m}^{2} / \mathrm{m}^{3}\right)\end{array}$ \\
\hline$C$ & $\begin{array}{l}\text { concentration of counter ions in the solution } \\
(\text { meq./l) }\end{array}$ \\
\hline$C^{*}$ & $\begin{array}{l}\text { equilibrium liquid phase concentration with } \\
\text { respect to resin phase (meq./l) }\end{array}$ \\
\hline$D$ & self-diffusion coefficient in solution $\left(\mathrm{cm}^{2} / \mathrm{s}\right)$ \\
\hline$D_{\text {eff }}$ & effective diffusivity $\left(\mathrm{cm}^{2} / \mathrm{s}\right)$ \\
\hline$K_{\mathrm{f}}$ & $\begin{array}{l}\text { rate parameter for film diffusion rate } \\
\text { equation }\left(\mathrm{s}^{-1}\right)\end{array}$ \\
\hline$L$ & resin bed height $(\mathrm{cm})$ \\
\hline$q$ & $\begin{array}{l}\text { concentration of one species of counter ions } \\
\text { in the resin (meq. } / \mathrm{ml})\end{array}$ \\
\hline$Q_{\mathrm{c}}$ & exchange capacity of the resin (meq./ml) \\
\hline$r_{0}$ & resin bead radius $(\mathrm{cm})$ \\
\hline$t$ & absolute time (s) \\
\hline$T$ & throughput parameter \\
\hline$u$ & linear flow rate $(\mathrm{cm} / \mathrm{s})$ \\
\hline$y$ & $\begin{array}{l}\text { normalized resin concentration of one of the } \\
\text { species of counter ions }\end{array}$ \\
\hline$z$ & $\begin{array}{l}\text { axial distance from the inlet of the resin } \\
\text { bed }(\mathrm{cm})\end{array}$ \\
\hline \multicolumn{2}{|c|}{ Greek symbols } \\
\hline$\alpha$ & separation factor \\
\hline$\beta$ & void fraction of resin bed \\
\hline$\delta$ & liquid film thickness $(\mathrm{cm})$ \\
\hline$\kappa$ & selectivity coefficient \\
\hline
\end{tabular}

Since the exchange of anionic species is accompanied by neutralization, the total anion or cation concentration is not constant. The value of $C_{i}^{*}$ thus depends on the variation of cation concentration, that is, the change of concentration of hydrogen ion. For the $\mathrm{Cl}^{-}-\mathrm{OH}^{-}$exchange system, the separation factor is defined by

$\alpha_{3}^{1}=\frac{\left[q_{1}\right]\left[C_{3}\right]}{\left[q_{3}\right]\left[C_{1}\right]}$

where $q$ and $C$ represent the concentration in resin and liquid phases, respectively. Subscript 1 denotes chloride ions and subscript 3 the hydroxide ions. For this binary exchange system, the equilibrium relation can also be expressed by

$\alpha_{3}^{1}=\frac{y\left[\mathrm{OH}^{-}\right]}{(1-y)\left[\mathrm{Cl}^{-}\right]}$

where $y$ is the equivalent fraction of exchanger in the chloride form. Since electroneutrality must be obeyed

$\left[\mathrm{Cl}^{-}\right]=\left[\mathrm{H}^{+}\right]-\left[\mathrm{OH}^{-}\right]=\frac{y\left[\mathrm{OH}^{-}\right]}{(1-y) \alpha_{3}^{1}}$

Assuming that $\left[\mathrm{OH}^{-}\right]$is negligible compared to $\left[\mathrm{H}^{+}\right]$and after substituting the dissociation constant for water, the equilibrium value of $\left[\mathrm{Cl}^{-}\right]$is

$\left[\mathrm{Cl}^{-*}\right]=0.0000001 \sqrt{\frac{y}{(1-y) \alpha_{3}^{1}}}$

Similarly, the equilibrium value for $\left[\mathrm{SO}_{4}{ }^{2-}\right]$ is

$\left[\mathrm{SO}_{4}{ }^{2-*}\right]=2.32 \times 10^{-10}\left[\frac{2 y}{\kappa_{3}^{2} Q_{\mathrm{c}}(1-y)}\right]^{1 / 3}$

where $y$ is the equivalent fraction of exchanger in the sulfate form, $\kappa_{3}^{2}$ is the selectivity coefficient for the exchange between sulfate and hydroxide ions.

For the ternary $\mathrm{SO}_{4}{ }^{2-}-\mathrm{Cl}^{-}-\mathrm{OH}^{-}$exchange system, the equilibrium relations are obtained from the relations defined for binary $\mathrm{Cl}^{-}-\mathrm{OH}^{-}$and $\mathrm{SO}_{4}{ }^{2-}-\mathrm{OH}^{-}$. We have

$\alpha_{3}^{1}=\frac{y_{1}\left[\mathrm{OH}^{-}\right]}{y_{3} C_{1}^{*}}$

$\kappa_{3}^{2} Q_{\mathrm{c}}=\frac{y_{2}\left[\mathrm{OH}^{-}\right]^{2}}{y_{3}^{2} C_{2}^{*}}$

Electroneutrality requires that

$C_{1}^{*}+C_{2}^{*}+\left[\mathrm{OH}^{-}\right]=\left[\mathrm{H}^{+}\right]=\frac{K_{\mathrm{w}}}{\left[\mathrm{OH}^{-}\right]}$

Given the resin phase composition $y_{1}$ and $y_{2}$, the values of $C_{1}^{*}, C_{2}^{*}$ and $\left[\mathrm{OH}^{-}\right]$can be determined by the above three independent equations.

For a feed solution containing multiple anionic species, a pseudo-ternary model can be used to characterize the exchange of these species with the hydroxide ions. In this case, all the divalent and monovalent species can be lumped together into two single components. An average $\alpha$ and $\kappa$ can be used for the calculation. The rate parameter $\left(K_{\mathrm{f}}\right)$ in the linear driving force film diffusion equation can be estimated by $K_{\mathrm{f}}=1.5 D_{\text {eff }} /\left(r_{0} \delta\right)$ [12], where $D_{\text {eff }}$ is the effective diffusivity in the liquid phase, $r_{0}$ the resin bead radius and $\delta$ the liquid film thickness. The liquid film thickness can be calculated from Glueckauf's correlation equation [12]. Helfferich $[13,14]$ showed that for ion exchange process involving neutralization, the rate actually depends on the diffusion of both the counter ions and the co-ions. The effective diffusivity for the $\mathrm{Cl}^{-}$and $\mathrm{OH}^{-}$exchange is derived by equating the net flux of $\mathrm{H}^{+}$and $\mathrm{Cl}^{-}$to obtain

$D_{\text {eff }}=\frac{2 D_{\mathrm{H}^{+}} D_{\mathrm{Cl}^{-}}}{D_{\mathrm{H}^{+}}+D_{\mathrm{Cl}^{-}}}$

Similarly, the effective diffusivity for the $\mathrm{SO}_{4}{ }^{2-}-\mathrm{OH}^{-}$exchange can be derived in the same manner to obtain

$D_{\text {eff }}=\frac{3 D_{\mathrm{H}^{+}} D_{\mathrm{SO}_{4}{ }^{2-}}}{D_{\mathrm{H}^{+}}+2 D_{\mathrm{SO}_{4}{ }^{2-}}}$

\section{Simulation of column performance}

Numerical algorithms were developed for the simulation of deanionization of the systems based on both binary and 
ternary models. The equations employed in the simulation were Eqs. (1) and (2), and the appropriate equilibrium relationships given in Eqs. (3)-(10). Simple boundary conditions of constant feed concentration and zero pre-saturation in the bed are also incorporated in the simulation. If needed, the numerical algorithms can be modified to take account of variable feed and non-uniform pre-saturation which are likely to occur in a cyclic process. The present experimental work covered $\mathrm{Cl}^{-}-\mathrm{OH}^{-}, \mathrm{SO}_{4}{ }^{2-}-\mathrm{OH}^{-}$and $\mathrm{SO}_{4}{ }^{2-}-\mathrm{Cl}^{-}-\mathrm{OH}^{-}$ exchange. The ion exchange in these three systems is accompanied by a neutralization reaction. The basic equations for computing column dynamics, which include continuity, rate and equilibrium relationship, are the same as those formulated for the ordinary exchange system. However, because of the neutralization, the total electrolyte concentration in the solution is not constant as in the case for the ordinary exchange system. Thus, the charge balance and the dissociation expression for water are also incorporated in the simulation calculation. The computations for the binary system of $\mathrm{Cl}^{-}-\mathrm{OH}^{-}$and $\mathrm{SO}_{4}{ }^{2-}-\mathrm{OH}^{-}$are easier to perform compared to the simulation for the ternary system. In the simulation for the ternary system, it is assumed that feed contains chloride and sulfate ions while the resin bed is initially in $\mathrm{OH}^{-}$form. The exchange and reaction between these three counter ions and the hydrogen co-ion proceed in two stages. In the first stage, all the $\mathrm{OH}^{-}$ions in the resin are being replaced by the incoming $\mathrm{Cl}^{-}$and $\mathrm{SO}_{4}{ }^{2-}$ ions in the solution. The $\mathrm{OH}^{-}$ ions released from the resin is further neutralized by $\mathrm{H}^{+}$ co-ion present in the solution. The second stage commences when all $\mathrm{OH}^{-}$ions in the resin are consumed and further treatment of feed solution will result in a redistribution of chloride and sulfate composition in the resin phase. For the binary and ternary system simulation, the self-diffusivities of $\mathrm{SO}_{4}{ }^{2-}, \mathrm{Cl}^{-}, \mathrm{OH}^{-}$and $\mathrm{H}^{+}$are taken from [15]. For binary system the effective diffusivity for $\mathrm{Cl}^{-}-\mathrm{OH}^{-}$and $\mathrm{SO}_{4}{ }^{2-}-\mathrm{OH}^{-}$exchange are calculated from Eqs. (11) and (12), respectively. For the ternary system simulation, the rate parameters in the two rate equations are assumed to be equal in the first stage of exchange. That is, assuming the effective diffusivity involving exchange between $\mathrm{Cl}^{-}$and $\mathrm{OH}^{-}$is the same as that of exchange between $\mathrm{SO}_{4}{ }^{2-}$ and $\mathrm{OH}^{-}$. The value of this effective diffusivity was obtained from the average value of $D_{\mathrm{Cl}^{-}-\mathrm{OH}^{-}}$and $D_{\mathrm{SO}_{4}{ }^{2-}-\mathrm{OH}^{-}}$. In the second stage of exchange, which involves only $\mathrm{Cl}^{-}$ and $\mathrm{SO}_{4}{ }^{2-}$, the effective diffusivity is calculated from the Nernst-Planck equation [16]. By assuming an equal equivalent fraction of sulfate and chloride ions in the resin phase, that is, $y_{\mathrm{Cl}^{-}}=y_{\mathrm{SO}_{4}{ }^{2-}}=0.5$, the average effective diffusivity is

$$
D_{\mathrm{SO}_{4}{ }^{2-}-\mathrm{Cl}^{-}}=\frac{5 D_{\mathrm{Cl}^{-}} D_{\mathrm{SO}_{4}{ }^{2-}}}{D_{\mathrm{Cl}^{-}}+4 D_{\mathrm{SO}_{4}{ }^{2-}}}
$$

The values of equilibrium parameters of $\alpha_{\mathrm{OH}^{-}}^{\mathrm{Cl}^{-}}$and $\kappa_{\mathrm{OH}^{-}}^{\mathrm{SO}_{4}^{2-}}$ used for the simulation study were taken from the published data of Anderson [17].

\section{Experimental}

In each run, fresh Dowex 1 (20-50 mesh) resins in chloride form were weighted and dried in an oven. The weight of the dry resins was recorded. The resins were placed in a beaker containing distilled water. The resin solutions were then slowly introduced to a $1.5 \mathrm{~cm}$ i.d. glass column for the preparation of a resin bed. Two liters of $0.5 \mathrm{~N} \mathrm{NaOH}$ solution was passed through the bed at a flow rate of $25 \mathrm{ml} / \mathrm{h}$. After the elution, the bed was washed with 21 of distilled water. The eluted solution, which contained chloride ions, together with the discharged wash water was collected. Samples of the collected solutions were analyzed for total chloride content. From the results of the analysis, the bed capacity was calculated. To start a run, the prepared resin bed in $\mathrm{OH}^{-}$ form was first rinsed with distilled water before feed solutions of either pure $\mathrm{HCl}$, pure $\mathrm{H}_{2} \mathrm{SO}_{4}$ or mixed feed of $\mathrm{HCl}$ and $\mathrm{H}_{2} \mathrm{SO}_{4}$ were introduced to the top of the resin bed. The flow rate was pre-adjusted with proper settings in a MasterFlex pump. The flow rates employed in the study were 75 , 100 and $150 \mathrm{ml} / \mathrm{min}$. In the process of treating the feed solution, samples of effluent from the bed were collected at regular interval (either 10 or $20 \mathrm{~min}$ ). The volume of samples collected was about $100-200 \mathrm{ml}$. The experimental run was conducted until the measurement of effluent $\mathrm{pH}$ and anion concentration measurements were almost identical to those of the feed solution. The collected samples were analyzed for their $\mathrm{pH}$ value and the $\mathrm{Cl}^{-}$and $\mathrm{SO}_{4}{ }^{2-}$ concentration. The analysis of $\mathrm{Cl}^{-}$and $\mathrm{SO}_{4}{ }^{2-}$ was conducted using a HACH DR 2000 spectrophotometer.

\section{Discussion of results}

Experimental results were obtained for column runs with feed solution containing only $\mathrm{HCl}$ or $\mathrm{H}_{2} \mathrm{SO}_{4}$. The feed concentrations for these two runs are both about $0.005 \mathrm{~N}$. Two other experimental runs were also conducted for a mixed feed solution containing about $0.0025 \mathrm{~N}$ of $\mathrm{HCl}$ and $0.0025 \mathrm{~N}$ of $\mathrm{H}_{2} \mathrm{SO}_{4}$. In all these runs, a $1.5 \mathrm{~cm}$ i.d. glass column was employed. The resin bed height was $40 \mathrm{~cm}$. The total bed volume with resin in $\mathrm{OH}^{-}$form is about $70.6 \mathrm{ml}$. Although the model equations proposed can be used to predict both the solution and resin phase concentration history, only the effluent concentration breakthrough data were determined experimentally. The experimental determination of resin phase concentration profile development was not performed in this study because of the expected tedious laboratory work involved. However, the solid phase composition characteristics were examined through the results obtained from simulation.

\subsection{Binary system}

Fig. 1a is the experimental data and calculated breakthrough curve for feed solution containing $0.0051 \mathrm{~N} \mathrm{Cl}^{-}$with 


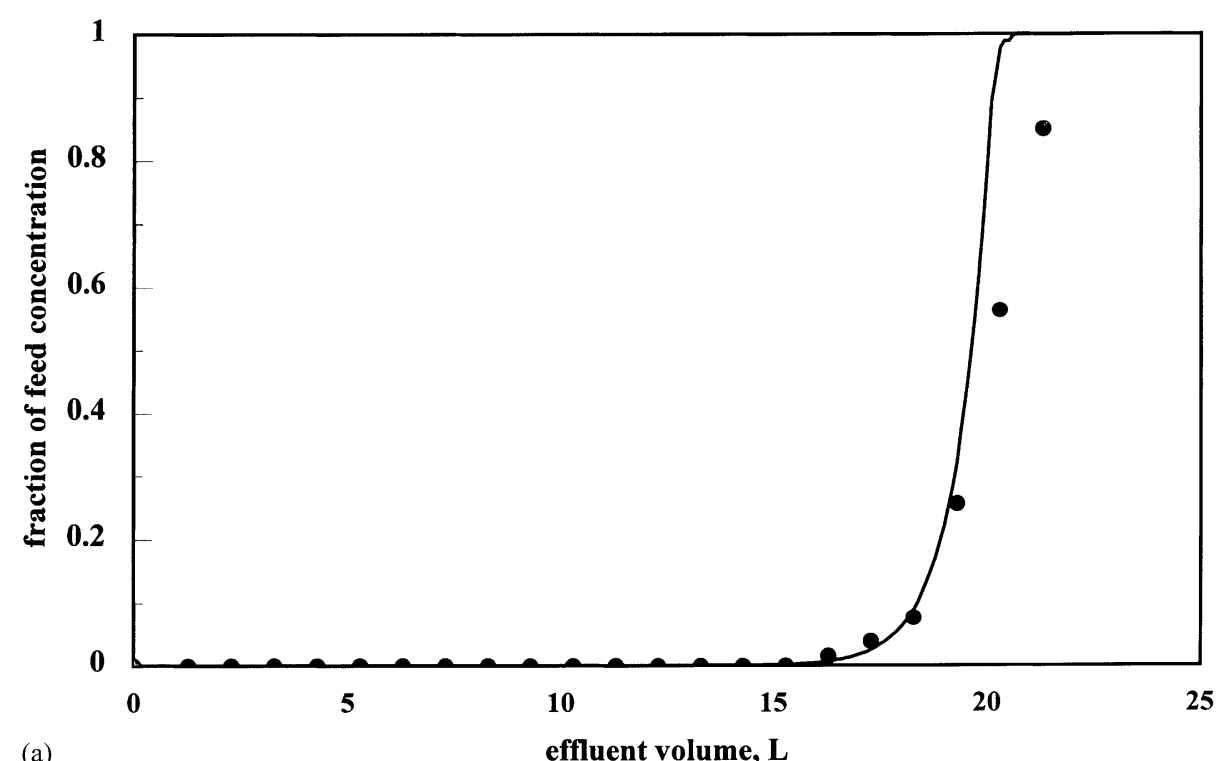

(a)

effluent volume, $L$

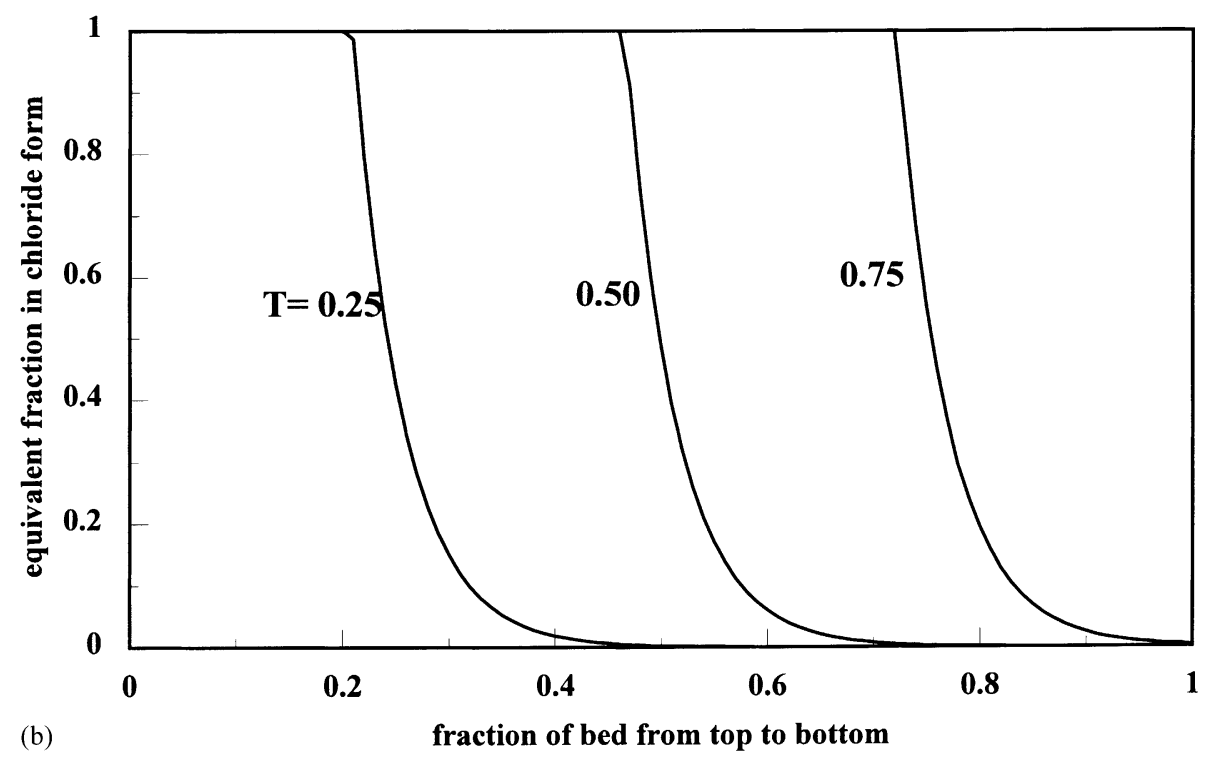

Fig. 1. (a) Experimental data and calculated breakthrough curve for $\mathrm{Cl}^{-}-\mathrm{OH}^{-}$exchange; (b) simulated resin phase concentration profile development for $\mathrm{Cl}^{-}-\mathrm{OH}^{-}$exchange.

a flow rate of $100 \mathrm{ml} / \mathrm{min}$. As mentioned before, Glueckauf [12] recommended the expression of $K_{\mathrm{f}}=1.5 D_{\text {eff }} /\left(r_{0} \delta\right)$ to calculate the rate parameter for film diffusion. The film thickness $(\delta)$ can be calculated from the correlation proposed by Glueckauf [12], in which $\delta=0.2 r_{0} /\left(1+70 u r_{0}\right)$, where $u$ is the linear flow rate in $\mathrm{cm} / \mathrm{s}, r_{0}$ the resin bead radius in $\mathrm{cm}$. For this run, the calculated effective diffusivity is $3.33 \times 10^{-5} \mathrm{~cm}^{2} / \mathrm{s}$. By assuming an average value of $r_{0}$ of $0.035 \mathrm{~cm}$ for the $20-50$ mesh resin used, the calculated film thickness from Glueckauf's correlation is $0.0021 \mathrm{~cm}$. Helfferich [18] stated that film thickness estimated from hydrodynamics consideration or kinetics measurements is about $0.001-0.01 \mathrm{~cm}$. The value of film thickness calculated is within this range. The calculated rate parameter $\left(K_{\mathrm{f}}\right)$ for the film diffusion rate equation is $0.68 \mathrm{~s}^{-1}$. The agreement between experimental and calculated breakthrough curve is satisfactory except for the later portion of the curve. The deviation is due to the contribution of particle diffusion that is expected when the bed is almost saturated with chloride ions. Fig. $1 \mathrm{~b}$ shows the simulated bed composition profile development for this run. The parameter $(T)$ employed in the figure is defined as the throughput ratio by Hiester and Vermeulen [19]. $T$ is a loading factor which represents the amount of ionic species loaded to the bed relative to the total resin bed capacity. It is seen that the bed profile is very sharp and that the reaction zone is very narrow. Fig. 2a is the experimental data and calculated breakthrough curve for a feed solution of $0.0052 \mathrm{~N} \mathrm{H}_{2} \mathrm{SO}_{4}$. Like the run for $\mathrm{Cl}^{-}-\mathrm{OH}^{-}$ 

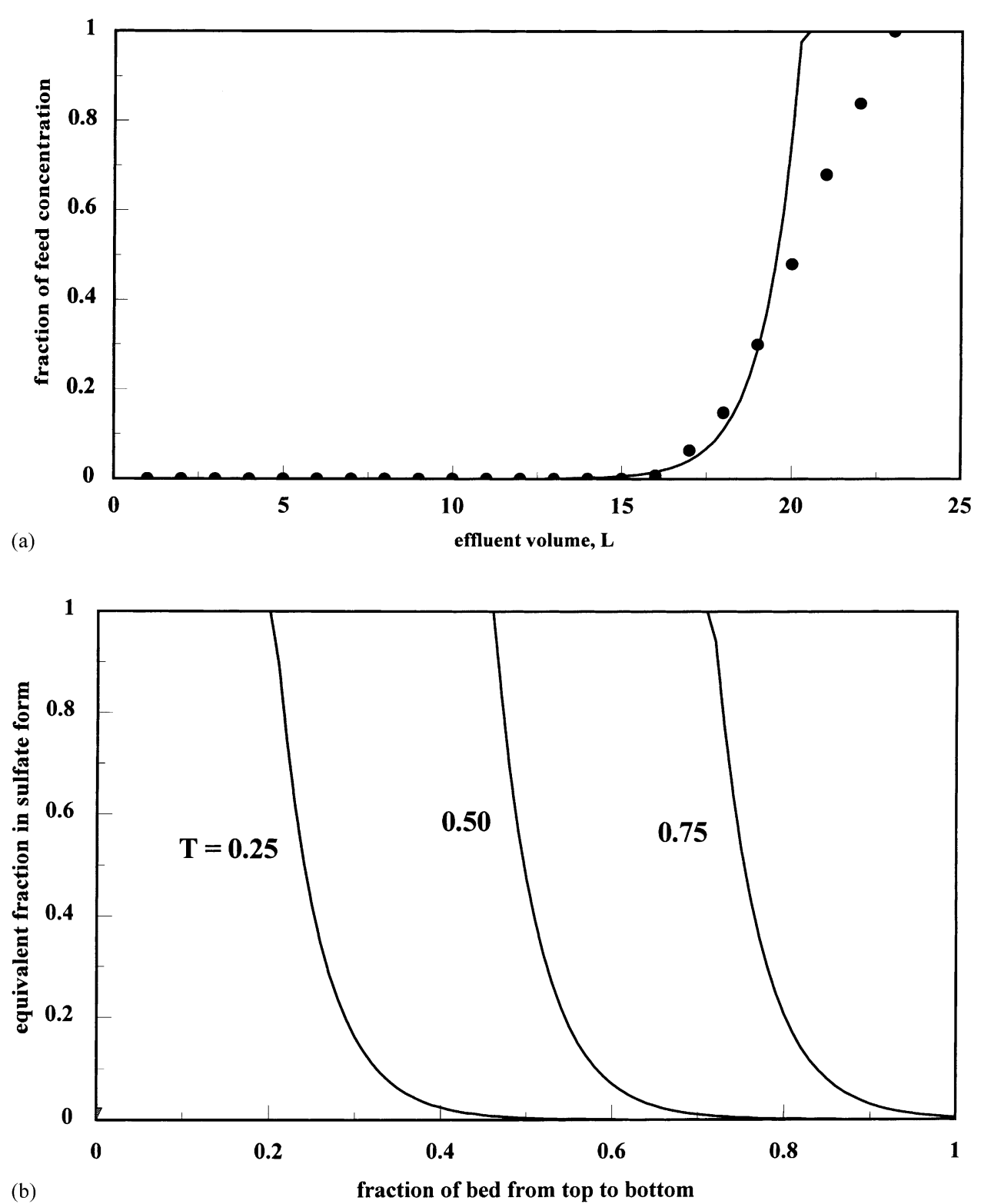

Fig. 2. (a) Experimental data and calculated breakthrough curve for $\mathrm{SO}_{4}{ }^{2-}-\mathrm{OH}^{-}$exchange; (b) simulated resin phase concentration profile development for $\mathrm{SO}_{4}{ }^{2-}-\mathrm{OH}^{-}$exchange.

exchange, the agreement between calculated and experimental breakthrough curve is also very good except for the later part of the data. For this run, the flow rate was also maintained at $100 \mathrm{ml} / \mathrm{min}$. Fig. $2 \mathrm{~b}$ shows simulated bed composition profile development for this particular run. Again, like Fig. 1b, a constant pattern [20] is also observed for the simulated results. The diffusivity used for the simulation of this run is $D_{\text {eff }}=2.56 \times 10^{-5} \mathrm{~cm}^{2} / \mathrm{s}$ and the calculated $K_{\mathrm{f}}$ is $0.53 \mathrm{~s}^{-1}$. Results of a graphical integration, over the entire breakthrough data shown in both Figs. 1a and 2a, agree with total bed capacity to within $3 \%$. This means that the mass balance for the counter ion is accurate within the range of the experimental error. The results of the two breakthroughs show that the curves are very sharp due to the irreversible exchange nature of $\mathrm{Cl}^{-}$and $\mathrm{SO}_{4}{ }^{2-}$ ions with $\mathrm{OH}^{-}$ions as a result of the neutralization reaction. The slight variation of $K_{\mathrm{f}}$ values used in the calculations does not result in any significant difference in the shape of breakthrough curves. It is expected that the breakthrough curves will be more diffuse if only ordinary exchanges are involved. Because of the very high rate of conversion of $\mathrm{OH}^{-}$form of resin to chloride or sulfate form, the degree of utilization is also very high. As revealed in Figs. 1a and 2a, at a breakthrough of $1 \%$, the degree of resin utilization is about $90 \%$. The bed profiles demonstrate the development of a constant pattern behavior, indicating a constant pattern model solution is applicable for simplifying quantitative study if desired. 


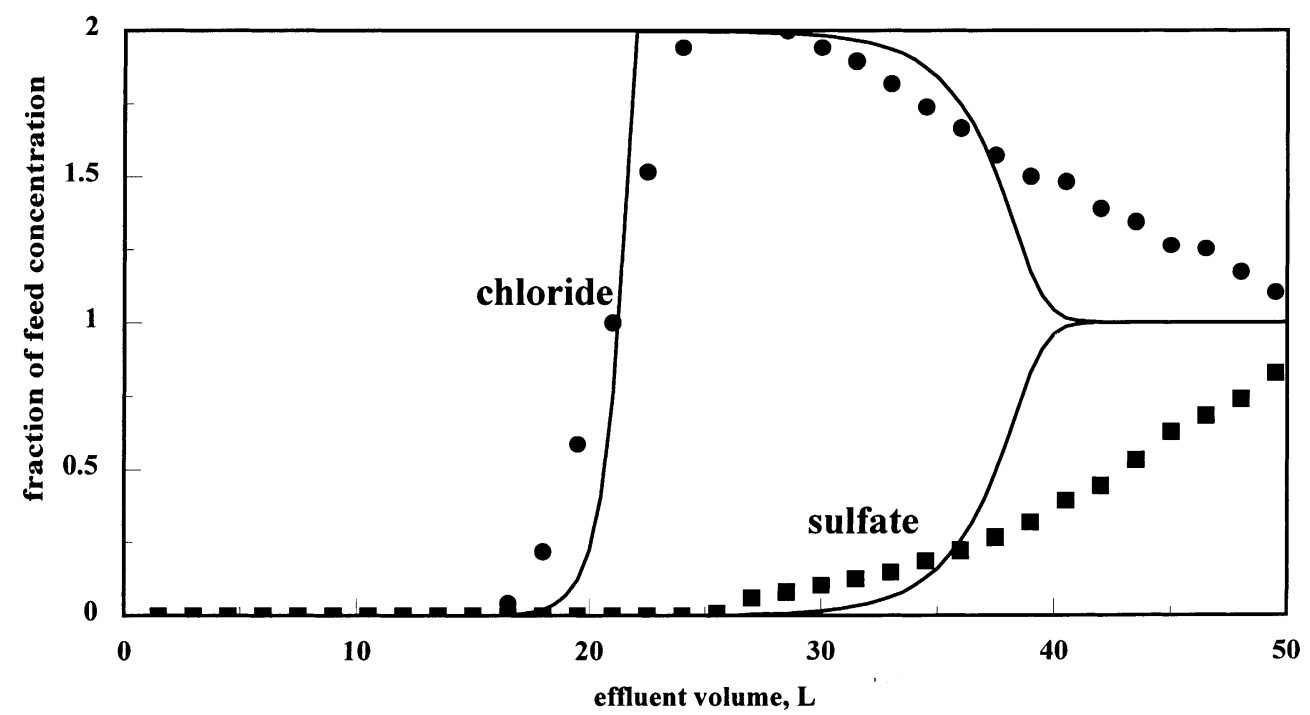

Fig. 3. Experimental data and calculated breakthrough curves for $\mathrm{SO}_{4}{ }^{2-}-\mathrm{Cl}^{-}-\mathrm{OH}^{-}$exchange, at flow rate of $75 \mathrm{ml} / \mathrm{min}$.

\subsection{Ternary system}

Two experimental column runs were performed with feed solutions containing a mixture of $\mathrm{HCl}$ and $\mathrm{H}_{2} \mathrm{SO}_{4}$. The total concentration in the feed was about $0.005 \mathrm{~N}$. The equivalent fractions of $\mathrm{HCl}$ and $\mathrm{H}_{2} \mathrm{SO}_{4}$ in the mixture were approximately equal to each other. The results of these two runs are shown in Figs. 3 and 4. The corresponding flow rates for these two runs are 75 and $150 \mathrm{ml} / \mathrm{min}$. In the simulation using ternary model, it is assumed that only $\mathrm{SO}_{4}{ }^{2-}, \mathrm{Cl}^{-}$ and $\mathrm{OH}^{-}$are involved in the anion exchange. The presence of $\mathrm{HSO}_{4}{ }^{-}$is expected to be negligible owing to neutralization and the high preference of resins for divalent $\mathrm{SO}_{4}{ }^{2-}$. For the experimental runs with mixed $\mathrm{HCl}$ and $\mathrm{H}_{2} \mathrm{SO}_{4}$ solution, the total resin bed capacity is determined to be about 104 meq. Figs. 3 and 4 show that the breakthrough of $\mathrm{Cl}^{-}$ ion raises to a maximum value, which exceeds its feed concentration, before falling back to the feed condition. This rollback behavior is typical in a multi-component ion exchange system in which the less preferred ions are being eluted out by the more preferred ionic species. When the total effluent concentration is plotted versus effluent volume, the breakthrough curve is also very sharp as in the case of treating either $\mathrm{HCl}$ or $\mathrm{H}_{2} \mathrm{SO}_{4}$ feed. The column behavior of this two-component feed system, with the bed initially saturated with the third component, can best be explained in terms of a two stages of exchange. In the first stage, the $\mathrm{OH}^{-}$in the resin has no selectivity with respect to the incoming anions in the solution. All the $\mathrm{Cl}^{-}$and $\mathrm{SO}_{4}{ }^{2-}$ ions are rapidly captured to the resin bed and $\mathrm{OH}^{-}$ions expelled from resin phase. The released $\mathrm{OH}^{-}$ions are subsequently being neutralized by hydrogen ions in the solution. When

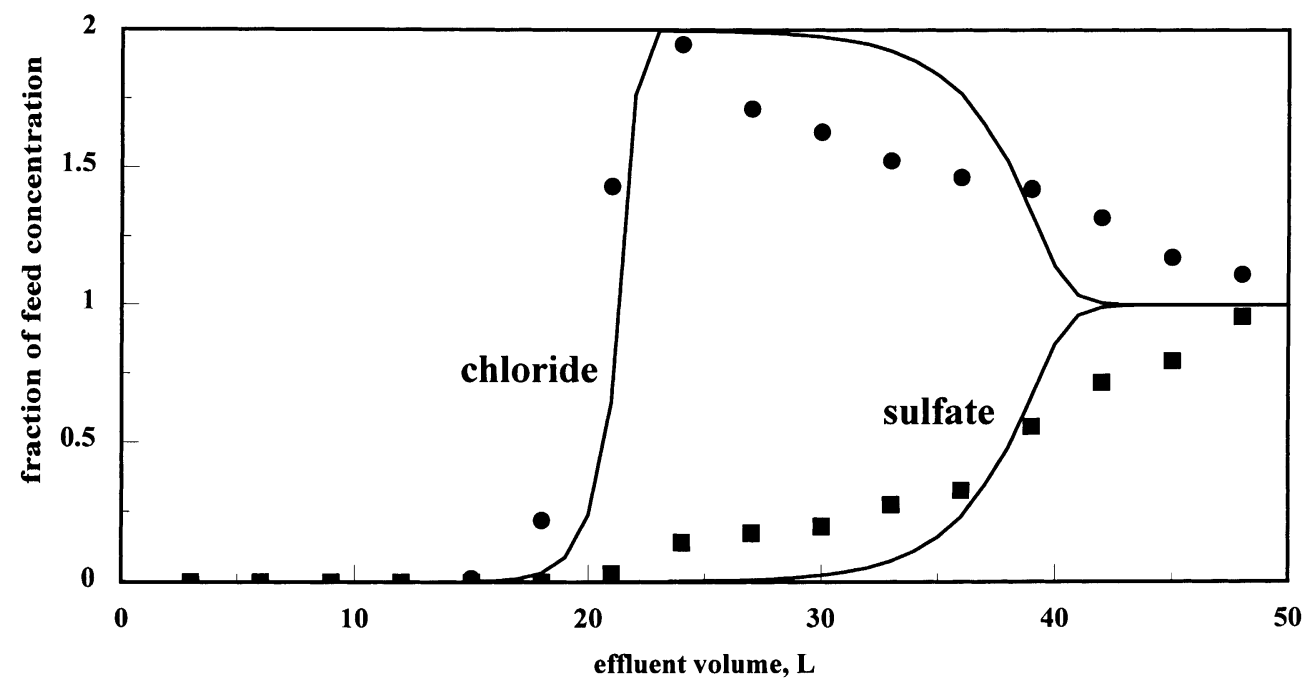

Fig. 4. Experimental data and calculated breakthrough curves for $\mathrm{SO}_{4}{ }^{2-}-\mathrm{Cl}^{-}-\mathrm{OH}^{-}$exchange, at flow rate of $150 \mathrm{ml} / \mathrm{min}$. 


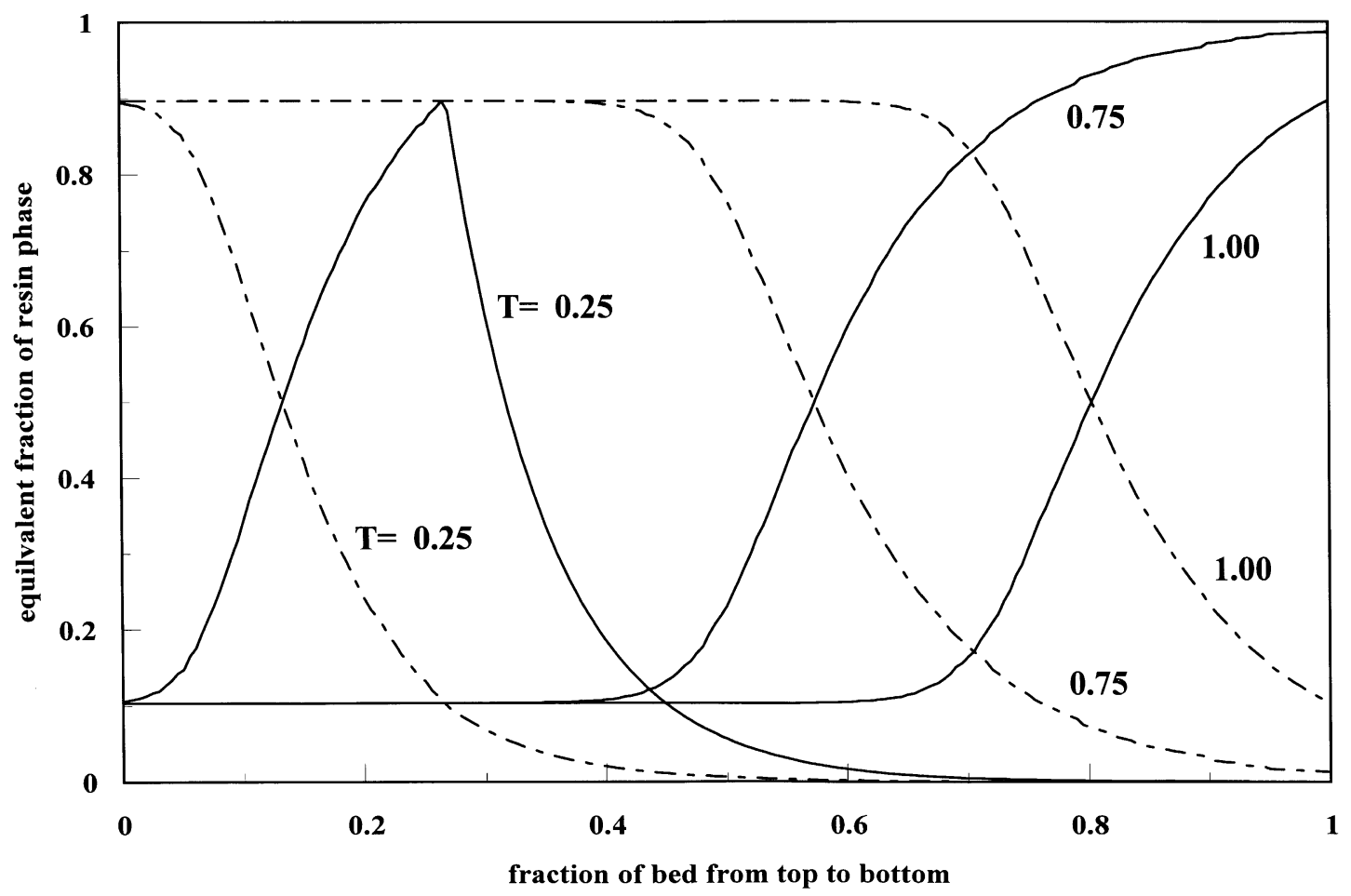

Fig. 5. Simulated resin phase concentration profile development for $\mathrm{SO}_{4}{ }^{2-}-\mathrm{Cl}^{-}-\mathrm{OH}^{-}$exchange (solid lines for chloride ion; dashed lines for sulfate ion).

the $\mathrm{OH}^{-}$ions in the resin phase are exhausted, an ordinary exchange of a binary system of $\mathrm{Cl}^{-}$and $\mathrm{SO}_{4}{ }^{2-}$ takes place. The bed composition is then readjusted and redistributed according to the $\mathrm{Cl}^{-}$and $\mathrm{SO}_{4}{ }^{2-}$ equilibrium relationship. The results show that the flow rate variation does not affect the time of breakthrough very significantly for chloride. However, the breakthrough for sulfate does come much earlier if a higher flow rate of $150 \mathrm{ml} / \mathrm{min}$ is used. The simulated results for these two runs are also shown in Figs. 3 and 4. Like the runs with a single type of acid. The agreement between experimental and simulated results are very satisfactory except for the later part of breakthrough curve. The reason for the discrepancy in the last part of breakthrough curve is likely due to the use of a constant value of effective diffusivity and in neglecting the effect of diffusion in the resin phase. To perform the simulation for the ternary system, the rate parameters must be defined or estimated. In this study, the physical properties and kinetic parameters for the rate equations are estimated from data available in literature or from published empirical correlation. The mass transfer coefficient in the film diffusion rate equation depends on the effective diffusivity, film thickness and resin beads radius. The values of film thickness were also calculated from Glueckauf's correlation. For flow rates of 75 and $150 \mathrm{ml} / \mathrm{min}$, the corresponding calculated values of $\delta / r_{0}$ were 0.073 and 0.0447 , respectively. The effective diffusivity for the first stage of exchange is assumed to be the same for the two runs with a value of $2.9 \times 10^{-5} \mathrm{~cm}^{2} / \mathrm{s}$. For the second stage of exchange involving only the exchange between chloride and sulfate ions, the effective diffusivity was calculated to be $1.72 \times 10^{-5} \mathrm{~cm}^{2} / \mathrm{s}$. For the first stage of exchange, the calculated values of $K_{\mathrm{f}}$ for the two experimental runs were 0.486 and $0.794 \mathrm{~s}^{-1}$. The normalized bed height parameter, which is defined by $K_{\mathrm{f}} L / u$, where $L$ is the bed height, is calculated to be 27.5 and 22.4. Fig. 5 is the simulated results of a typical bed profile development in which the displacement effect of the divalent ion on monovalent ion is clearly shown. It should be emphasized that the rate parameters used in the simulation were estimated independently from resin bed experimental data. This is in contrast with many theoretical studies in which the rate parameters were obtained by fitting experimental breakthrough curves. Since the purpose of the deionization is the removal of all the ions present, a simplified model that can predict the breakthrough of any ion is very useful. The use of a pseudo-ternary model can facilitate the design calculation of the anion bed performance in a two-bed deionization process.

\section{Summary and conclusions}

Experiments and simulations were performed for deionization of dilute $\mathrm{HCl}$ and $\mathrm{H}_{2} \mathrm{SO}_{4}$ by ion exchange with $\mathrm{OH}^{-}$ form resin in an anion resin bed. The experimental results indicate that $\mathrm{OH}^{-}$in the resin has no selectivity toward chloride or sulfate ion in the solution. The exchange with the $\mathrm{OH}^{-}$ion is irreversible and is not significantly affected by the equilibrium relationships with the counter ions. When 
only $\mathrm{HCl}$ or $\mathrm{H}_{2} \mathrm{SO}_{4}$ is treated in the resin bed, the breakthrough curves for $\mathrm{Cl}^{-}$or $\mathrm{SO}_{4}{ }^{2-}$ are steep and sharp, which leads to very high degree of resin utilization. Results of simulation study also indicated a constant pattern for the bed composition profile development. The ternary model developed for simulation can be extended to a multiple species system in which various monovalent and divalent species are present.

\section{Acknowledgements}

The financial support by the National Science Council of Republic of China is gratefully acknowledged.

\section{References}

[1] M.C. Gottieb, P. Meyers, in: Proceedings of the Presentation of the Paper at Ion Exchange at the Millennium 2000, Cambridge, 16-21 July 2000.

[2] F.J. DeSilva, in: Proceedings of the Presentation of the Paper at 25th Annual WQA Conference, 1999.

[3] B. Applebaum, Demineralization by Ion Exchange, Academic Press, New York, 1968 (Chapter 6).
[4] V. Chowdiah, G.L. Foutch, Ind. Eng. Chem. Res. 34 (1995) 4040.

[5] V. Chowdiah, G.L. Foutch, in: J.A. Greig (Ed.), Ion Exchange Developments and Applications, The Royal Society of Chemistry, Cambridge, 1996, p. 10.

[6] G.E. Haub, G.L. Foutch, Ind. Eng. Chem. Fundam. 25 (1986) 338

[7] E.J. Zecchini, G.L. Foutch, Ind. Eng. Chem. Res. 30 (1991) 1886.

[8] T.V. Arden, in: K. Dorfner (Ed.), Ion Exchangers, Walter de Gruyter, New York, 1991, p. 717.

[9] F.X. McGarvey, in: J.A. Greig (Ed.), Ion Exchange Developments and Applications, The Royal Society of Chemistry, Cambridge, 1996, p. 82.

[10] F.X. McGarvey, D. Tamaki, in: M. Abe, T. Kataoka, T. Suzuki (Eds.) New Developments in Ion Exchange, Kodansha, Tokyo, 1991, p. 331.

[11] F. Helfferich, Y.L. Hwang, in: K. Dorfner (Ed.), Ion Exchangers, Walter de Gruyter, New York, 1991, p. 1277.

[12] E. Glueckauf, Ion Exchange and Its Applications, Society of Chemical Industry, London, 1955, p. 34.

[13] F. Helfferich, J. Phys. Chem. 69 (1965) 1178.

[14] F. Helfferich, in: L.A. Marinsky (Ed.), Ion Exchange, Marcel Dekker, New York, 1966 (Chapter 2).

[15] E.L. Cussler, Diffusion, Mass Transfer in Fluid System, Cambridge University Press, Cambridge, 1984.

[16] F. Helfferich, M.S. Plesset, J. Chem. Phys. 28 (1958) 418.

[17] R.E. Anderson, AIChE Symp. Ser. 71 (1975) 152.

[18] F. Helfferich, Ion Exchange, McGraw-Hill, New York, 1962 (Chapter 6).

[19] N.K. Hiester, T. Vermeulen, Chem. Eng. Prog. 48 (1952) 505.

[20] A.S. Michaels, Ind. Eng. Chem. 44 (1952) 1922. 